\title{
PICK1 and Phosphorylation of the Glutamate Receptor 2 (GluR2) AMPA Receptor Subunit Regulates GluR2 Recycling after NMDA Receptor-Induced Internalization
}

\author{
Da-Ting Lin and Richard L. Huganir \\ Department of Neuroscience, Johns Hopkins University School of Medicine, Howard Hughes Medical Institute, Baltimore, Maryland 21205
}

\begin{abstract}
Changes in surface trafficking of AMPA receptors play an important role in synaptic plasticity. Phosphorylation of the $\mathrm{C}$ terminus of the AMPA receptor (AMPAR) subunit glutamate receptor 2 (GluR2) and the binding of GluR2 to the PDZ [postsynaptic density-95/Discs large/zona occludens-1]-domain containing protein, protein interacting with protein kinase C (PICK1), have been proposed to play an important role in NMDA receptor dependent internalization of GluR2. However, the fate of internalized GluR2 after NMDA receptor (NMDAR) activation is still unclear. Both recycling and degradation of GluR2 after the activation of NMDAR have been reported. Here, we used a pH-sensitive green fluorescent protein variant, pHluorin, tagged to the $\mathrm{N}$ terminus of GluR2 (pH-GluR2) to study the dynamic internalization and recycling of GluR2 after NMDAR activation. Using fluorescence recovery after photobleach (FRAP), we directly demonstrate that internalized pH-GluR2 subunits recycle back to the cell surface after NMDAR activation. We further demonstrate that changing the phosphorylation state of the S880 residue at the C terminus of GluR2 does not affect NMDAR-dependent GluR2 internalization, but alters the recycling of GluR2 after NMDAR activation. In addition, mutation of the $\mathrm{N}$-ethylmaleimide-sensitive fusion protein (NSF) binding site in the pH-GluR2 slows receptor recycling. Finally, neurons lacking PICK1 display normal NMDAR dependent GluR2 internalization compared with wild-type neurons, but demonstrate accelerated GluR2 recycling after NMDAR activation. These results indicate that phosphorylation of GluR2 S880 and NSF and PICK1 binding to GluR2 dynamically regulate GluR2 recycling.
\end{abstract}

Key words: AMPA receptor; endocytosis; phosphorylation; PICK1; NSF; FRAP

\section{Introduction}

AMPA receptors (AMPARs) and NMDA receptors (NMDARs) are the major excitatory neurotransmitter receptors in the CNS. AMPARs mediate the majority of fast excitatory synaptic transmission while NMDARs play an essential role in the induction of certain forms of synaptic plasticity (Hollmann and Heinemann, 1994; Dingledine et al., 1999). The regulation of the dynamic membrane trafficking of AMPARs plays a critical role in controlling the levels of synaptic AMPARs and in the expression of several forms of synaptic plasticity such as long-term potentiation (LTP) and long-term depression (LTD). A variety of studies have demonstrated that phosphorylation of AMPARs and AMPAR interacting proteins dynamically regulate receptor trafficking and the expression of LTP and LTD (Song and Huganir, 2002). Several studies have shown that the AMPAR interacting protein PICK1 regulates the surface expression of glutamate receptor 2 (GluR2) (Perez et al., 2001; Terashima et al., 2004) and disrupting

\footnotetext{
Received June 15, 2006; revised Nov. 1, 2007; accepted Nov. 13, 2007.

This work was supported by National Institutes of Health Grant NS036715 and the Howard Hughes Medical Institute.

We thank Dr. J. E. Rothman for providing pHluorin coding CDNA, Jordan Steinberg for genotyping PICK1 knockout mice, and Drs. Yun Li, Hengye Man, Jean-Claude Beique, and Gareth M. Thomas for critical reading of this manuscript.

Correspondence should be addressed to Richard L. Huganir at the above address. E-mail: rhuganir@jhmi.edu. DOI:10.1523/JNEUROSCI.1750-07.2007

Copyright $\odot 2007$ Society for Neuroscience $\quad$ 0270-6474/07/2713903-06\$15.00/0
}

GluR2-PICK1 interactions blocks the expression of LTD (Xia et al., 2000; Iwakura et al., 2001; Kim et al., 2001; Chung et al., 2003). In contrast, the AMPAR interacting protein $\mathrm{N}$-ethylmaleimide-sensitive fusion protein (NSF) increases the surface expression of GluR2 (Huang et al., 2005). Disrupting NSF binding to GluR2 decreases AMPAR mediated synaptic transmission (Nishimune et al., 1998; Song et al., 1998) and the surface expression of GluR2 (Noel et al., 1999). NSF has been proposed to regulate GluR2 surface expression by disassembling GluR2PICK1 complexes (Hanley et al., 2002). Interestingly, phosphorylation of serine 880 (S880) within the C terminus of the GluR2 subunit differentially regulates the interaction of GluR2 with two postsynaptic density-95/Discs large/zona occludens-1 (PDZ)domain containing proteins, glutamate receptor interacting protein 1/2 (GRIP1/2) and PICK1, and modulates the membrane trafficking of GluR2 and the expression of LTD (Xia et al., 2000; Chung et al., 2003; Seidenman et al., 2003; Steinberg et al., 2006). Together, these data indicate that interaction of PICK1 with GluR2 either promotes internalization or inhibits recycling of GluR2 and retains GluR2 intracellularly. The precise role of PICK1 in regulating GluR2 trafficking, however, remains unclear.

To examine the regulation of AMPAR membrane trafficking in more detail we have used the $\mathrm{pH}$-sensitive green fluorescent protein, pHluorin (Miesenbock et al., 1998), fused to the $\mathrm{N}$ terminus of the GluR2 (pH-GluR2) to study AMPAR membrane 
trafficking with high temporal resolution (Ashby et al., 2004; Kopec et al., 2006). This construct can be used to monitor the endocytosis/exocytosis of GluR2 in response to NMDAR activation. By combining imaging $\mathrm{pH}-\mathrm{GluR} 2$ with fluorescence recovery after photobleach (FRAP), we demonstrated that after NMDAR activation, $\mathrm{pH}-\mathrm{GluR} 2$ recycled back to the surface. We further demonstrated that altering the phosphorylation states of GluR2 S880 residue as well as disrupting PICK1 binding to GluR2 did not impair NMDAR induced GluR2 internalization. Instead, alterations in this phosphorylation site resulted in changes in the rate of recycling of pH-GluR2 after NMDAR activation. Finally, we showed that in PICK1 knock-out hippocampal cultures, GluR2 recycling after NMDAR activation was accelerated although GluR2 internalization in response to NMDAR activation remained unchanged. These results indicate that phosphorylation of GluR2 S880, which differentially regulated PICK1 binding to GluR2, also regulates GluR2 recycling to the plasma membrane.

\section{Materials and Methods}

All chemicals were obtained from Fisher Scientific (Pittsburgh, PA) unless otherwise specified. All restriction enzymes were purchased from New England Biolabs (Ipswich, MA).

Super Ecliptic pHluorin coding sequence was amplified by PCR and inserted into the $\mathrm{N}$ terminus of GluR2 after signal peptide. To generate $\mathrm{C}$-terminal point mutants of $\mathrm{pH}-\mathrm{GluR} 2$, the C terminus of GluR2 S880E, K882A (Chung et al., 2003), and $\Delta$ NSF (Steinberg et al., 2004) were exchanged with that of $\mathrm{pH}-\mathrm{GluR} 2$ by subcloning. DNA sequences were verified by automated DNA sequencing in the Johns Hopkins University School of Medicine DNA sequencing Core facility. For details about hippocampal neuronal cultures and transfection and imaging and data analysis see the supplemental Methods (available at www.jneurosci.org as supplemental material).

\section{Results}

Use of pHluorin tagged GluR2 fusion proteins to analyze the membrane trafficking of the AMPAR GluR2 subunit after NMDAR activation

Ecliptic pHluorin has the distinctive feature that it is fluorescent at $\mathrm{pH} \sim 7.4$ and nonfluorescent at $\mathrm{pH}<6.0$ under $488 \mathrm{~nm}$ excitation. The $\mathrm{N}$ terminus of GluR2 is exposed to either the extracellular space ( $\mathrm{pH} \sim 7.4$ ) or the lumen of endosomes $(\mathrm{pH}<6.0)$ when the receptors reside on the plasma membrane or endosomes, respectively. The $\mathrm{pH}-\mathrm{GluR} 2$ construct was engineered such that pHluorin was inserted into the $\mathrm{N}$ terminus of GluR2 after the signal peptide. The change of pHluorin fluorescence can then be used as an indicator of receptor redistribution between plasma membrane and endosomal compartments after NMDAR activation (Ashby et al., 2004). When pH-GluR2 was expressed in
B

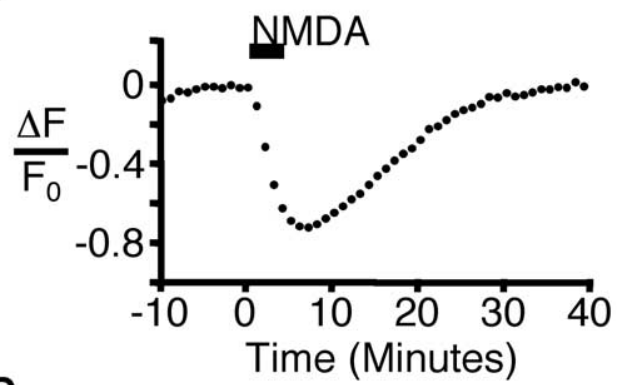

D

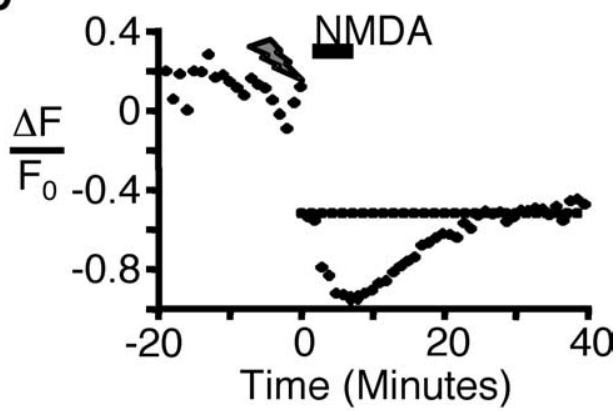

$\mathbf{F}$

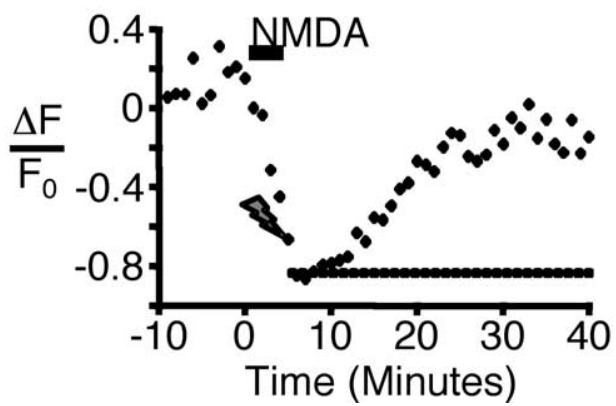

Figure 1. $\quad \mathrm{pH}-$-GluR2 is internalized following NMDAR activation and recycles to the cell surface. $\boldsymbol{A}$, Representative images of a hippocampal neuron subjected to NMDA perfusion/washout cycle show that NMDA induces loss of significant amount of observed $\mathrm{pH}-\mathrm{GluR2}$ fluorescence; washout of NMDA resulted in full return of $\mathrm{pH}-\mathrm{GluR2}$ fluorescence over time. $\boldsymbol{B}$, Time trace of pH-GluR2 列 ing pH-GluR2 shown (top left). pH-GluR2 on the surface of the cell soma was photobleached immediately before NMDA perfusion, 位 (unbleached area) and recovery of pH-GluR2 fluorescence to postbleached level in somatic surface (bleached area, white square) 列 作 (unbleached area) and almost complete recovery of pH-GluR2 fluorescence on the somatic surface (bleached area, white square). $\boldsymbol{F}$, Time trace of fluorescence change in somatic (bleached) surface for the experiment presented in $\boldsymbol{E}$. Scale bars: $\boldsymbol{A}, \boldsymbol{C}, \boldsymbol{E}, 10 \mu \mathrm{m}$.

hippocampal neurons, the majority of pH-GluR2 fluorescence originated from surface-exposed $\mathrm{pH}-\mathrm{GluR2}$ proteins and was sensitive to rapid changes in extracellular $\mathrm{pH}$ (supplemental Fig. $1 A$, available at www.jneurosci.org as supplemental material). Moreover, perfusion of NMDA for $5 \mathrm{~min}$, which is known to induce rapid internalization of GluR2, induced a significant loss of pH-GluR2 fluorescence. Interestingly, washout of NMDA resulted in full recovery of fluorescence within $30 \mathrm{~min}$ (Fig. $1 \mathrm{~A}, B$, supplemental movie 1 , available at www.jneurosci.org as supplemental material). This process could be repeated and the $\mathrm{pH}$ GluR2 demonstrated similar kinetics of loss/recovery of fluorescence over several cycles in both somatic and dendritic areas (supplemental Fig. $1 B$, available at www.jneurosci.org as supple- 
mental material). However, we did not observe significant change in fluorescence of dendritic $\mathrm{pH}-\mathrm{GluR} 2$ punta (most likely synaptic clusters of GluR2) in response to 5 min NMDAR activation over a $1 \mathrm{~h}$ period of recording. The fluorescence loss/recovery of $\mathrm{pH}$-GluR2 induced by NMDA perfusion/washout was fully blocked by APV, a specific NMDAR antagonist (supplemental Fig. $1 C$, available at www.jneurosci.org as supplemental material), suggesting that the change of pHluorin fluorescence required NMDAR activation. In addition, the change of pHluorin fluorescence in response to NMDA perfusion was highly sensitive to extracellular $\mathrm{Ca}^{2+}$ concentration (supplemental Fig. $1 D$, available at www.jneurosci.org as supplemental material), indicating that the change of fluorescence after NMDAR activation required calcium entry and was not likely caused by changes in $\mathrm{pH}$ values in either extracellular solutions or intracellular environments. Furthermore, this change of $\mathrm{pH}$-GluR2 fluorescence is fully blocked by two membrane-permeable dynamin inhibitory peptides, but not a membrane permeable control peptide (supplemental Fig. 2, available at www.jneurosci.org as supplemental material) (Brebner et al., 2005). Together, these data demonstrate that the fluorescence loss during NMDA perfusion represents NMDAR induced GluR2 internalization and the fluorescence recovery after NMDA washout represents insertion of pHluorin GluR2 into the plasma membrane.

To determine whether the inserted GluR2 after NMDAR induced internalization was caused by de novo insertion of new receptors or recycling of the internalized receptors, we combined imaging of $\mathrm{pH}-\mathrm{GluR} 2$ during NMDA perfusion/washout with FRAP. We reasoned that if the inserted receptors were from preexisting intracellular pools, NMDA perfusion/washout after photobleach should result in full recovery of $\mathrm{pH}$-GluR2 fluorescence, assuming that the nonfluorescent intracellular pools cannot be photobleached. Conversely, if the inserted receptors were those that were originally present on the surface and internalized by NMDAR activation, NMDA perfusion/washout after photobleach should result in recovery of $\mathrm{pH}-\mathrm{GluR} 2$ fluorescence only to the postphotobleach level, because of the fact that the reinserted receptors would carry a photobleached and nonfluorescent form of pHluorin. A hippocampal neuron expressing $\mathrm{pH}$ GluR2 before photobleach is shown in Figure $1 C$ (top left). The surface of the soma was chosen for photobleach to minimize possible contribution of lateral diffusion to the fluorescence recovery after photobleach. After photobleach, a significant proportion of $\mathrm{pH}-\mathrm{GluR} 2$ fluorescence was eliminated (Fig. 1C, top right). Subsequent perfusion of NMDA for 5 min induced GluR2 internalization in both the somatic and dendritic areas (Fig. $1 C$, bottom left). pH-GluR2 fluorescence in a unbleached area (dendritic surface) fully recovered to baseline level $30 \mathrm{~min}$ after NMDA washout (Fig. 1C, bottom right). However, pH-GluR2 fluorescence in the photobleached area (somatic surface) only recovered to the postphotobleach level (Fig. $1 C$, bottom right, $D$ ). This result suggests that after NMDAR activation, the inserted $\mathrm{pH}-\mathrm{GluR} 2$ was originated from recycled receptors that were originally present on the surface. However, an alternative explanation could be that the lack of full recovery of fluorescence was attributable to either photodamage to the cellular machinery responsible for receptor trafficking or photobleach of intracellular invisible receptor pools. To address this issue, a second FRAP experiment was performed in which the $\mathrm{pH}-\mathrm{GluR} 2$ was first relocated to intracellular pools by the activation of NMDA receptors and photobleach was then performed immediately before NMDA washout. We reasoned that if the photobleach protocol caused photodamage to the receptor trafficking machinery or photobleach of intracellular invisible $\mathrm{pH}-\mathrm{GluR} 2$ pools, the pho- tobleached area should also demonstrate a lack of recovery of pH-GluR2 fluorescence. Conversely, if the photobleach caused no damage to the trafficking machinery, a full recovery of fluorescence after NMDA washout should be observed. A hippocampal neuron expressing pH-GluR2 is shown in Figure $1 E$ (top left). After stimulation with NMDA for 5 min the majority of $\mathrm{pH}$ GluR2 fluorescence disappeared consistent with relocation of receptors to intracellular compartments (Fig. $1 E$, top right). The same photobleach protocol was then performed (Fig. $1 E$, bottom left). We observed a small amount of additional fluorescence loss in the bleached area, which is attributable to photobleach of the remaining surface $\mathrm{pH}-\mathrm{GluR} 2$. After photobleach, fluorescence of $\mathrm{pH}-\mathrm{GluR} 2$ fully recovered on the dendritic surface (nonbleached) and almost fully recovered on the somatic surface (photobleached) area (Fig. $1 E$, bottom right, $F$ ). The slight reduction in the fluorescence recovery on the somatic surface was likely caused by photobleach of remaining surface pH-GluR2 fluorescence during our photobleach protocol. Together, these two FRAP experiments demonstrate that the inserted pH-GluR2 after NMDAR activation is derived from recycled receptors that were internalized in response to NMDAR activation.

\section{Phosphorylation state of GluR2 S880 regulates GluR2 recycling}

The S880 residue in the C terminus of GluR2 has been shown to be a target site for PKC phosphorylation in vitro and in vivo (Chung et al., 2000). In addition, we showed that after NMDAR activation, both PKC activity and phosphorylation level of GluR2 S880 residue were transiently increased and returned to baseline after NMDA washout (supplemental Fig. 3, available at www. jneurosci.org as supplemental material), suggesting that NMDAR activation can regulate phosphorylation state of GluR2 S880 through activation of PKC pathway. A variety of data have shown that changes in the phosphorylation state of $\mathrm{S} 880$ residue regulates activity dependent GluR2 trafficking. However, it has been difficult to analyze this in detail using either biotinylation or immunostaining approaches. To examine the effect of changes in the $S 880$ residue phosphorylation state on the dynamics of GluR2 membrane trafficking in response to NMDAR activation, we generated two $\mathrm{pH}-\mathrm{GluR} 2$ point mutants. One mutant, $\mathrm{pH}-\mathrm{GluR} 2$ S880E, should mimic phosphorylation of this residue whereas the other mutant, pH-GluR2 K882A, should inhibit PKC phosphorylation of S880 without affecting PDZ domain interactions (Chung et al., 2000). Hippocampal neurons expressing either pH-GluR2 S880E or K882A are shown in Figure $2 \mathrm{~A}$. Perfusion of NMDA for $5 \mathrm{~min}$ induced internalization of $\mathrm{pH}-\mathrm{GluR} 2, \mathrm{pH}-$ GluR2 S880E, and pH-GluR2 K882A at levels of $73.8 \pm 1.6 \%$ $(n=19), 71.0 \pm 3.4 \%(n=5)$, and $68.0 \%+3.0 \%(n=4)$, respectively (Fig. $2 B, C$ ). There is no statistical difference in NMDAR activation induced GluR2 internalization between $\mathrm{pH}$ GluR2 wt and S880E ( $p=0.443, t$ test $)$ or pH-GluR2 wt and K882A ( $p=0.144, t$ test). However, interestingly, reinsertion of $\mathrm{pH}-$ GluR2 displayed a $T_{1 / 2}$ (see Materials and Methods) of $10.3 \pm$ $0.4 \min (n=19)$ after NMDA washout, whereas the reinsertion of $\mathrm{pH}-\mathrm{GluR} 2 \mathrm{~S} 880 \mathrm{E}$ was significantly prolonged $\left(T_{1 / 2}=16.8 \pm\right.$ $1.4 \mathrm{~min} ; n=5$; $p<0.001, t$ test) (Fig. $2 B, C$ ). In contrast, $\mathrm{pH}$ GluR2 K882A showed accelerated reinsertion after NMDA washout $\left(T_{1 / 2}=6.3 \pm 0.8 \mathrm{~min} ; n=4 ; p<0.001, t\right.$ test) (Fig. $\left.2 B, C\right)$. These results indicated that the change in the phosphorylation state of GluR2 S880 is not critical for NMDAR activation induced GluR2 internalization, but instead regulates the recycling of GluR2 after NMDAR activation. 
NSF affects GluR2 recycling after

NMDAR activation

NSF has been shown to dissociate the complex of GluR2 and PICK1 (Hanley et al., 2002), and this activity of NSF has been shown to facilitate synaptic targeting of GluR2-containing AMPARs (Steinberg et al., 2004; Gardner et al., 2005). To test whether NSF facilitates recycling of GluR2 after NMDAR activation, a pH-GluR2 mutant that eliminated the NSF binding site (pH-GluR2 $\Delta$ NSF: V848L, A849T, P852T point mutation) (Steinberg et al., 2004) was analyzed in the assay. A hippocampal neuron expressing $\mathrm{pH}-\mathrm{GluR} 2$ $\triangle$ NSF is shown in Figure $2 A$ (bottom). Perfusion of NMDA for $5 \mathrm{~min}$ induced $75.0 \pm 2.8 \%$ internalization of surface $\mathrm{pH}$ GluR2 $\Delta$ NSF $(n=6)$, similar to that of pH-GluR2 ( $p=0.731, t$ test). However, the recycling of pH-GluR2 $\Delta$ NSF was significantly prolonged after NMDA washout $\left(T_{1 / 2}=14.2 \pm 1.1 \mathrm{~min} ; n=6 ; p<0.001, t\right.$ test) (Fig. $2 B$, bottom, $C$ ). This suggests that NSF facilitates the recycling of GluR2 after NMDAR activation.

PICK1 knock-out results in accelerated GluR2 recycling process

To test the role of PICK1 in regulating GluR2 recycling after NMDAR activation, we examined pH-GluR2 internalization/ recycling in PICK1 knock-out neurons (Gardner et al., 2005). Hippocampal neurons were derived from postnatal day 0 (P0) wild-type or PICK1 knock-out mice and transfected with pH-GluR2 (Fig. 3A). NMDA perfusion for $5 \mathrm{~min}$ induced $61.8 \pm 3.2 \%$ of pH-GluR2 internalization in PICK1 knock-out neurons $(n=5)$, and $63.0 \pm 5.5 \%$ in neurons derived from wild-type mice ( $n=5 ; p=0.855, t$ test). This result suggests that PICK1 is not required for NMDA induced GluR2 internalization in hippocampal neurons (Fig. $3 B, C)$. However, after NMDA washout, PICK1 knock-out neurons demonstrated significant acceleration of GluR2 recycling $\left(T_{1 / 2}=4.8 \pm 0.4 \mathrm{~min} ; n=5\right)$ compared with control neurons $\left(T_{1 / 2}=11.6+0.9\right.$ min; $n=5 ; p<0.001, t$ test) (Fig. $3 B, C$ ). This result strongly suggests that, at least in hippocampal neurons, PICK1 retains internalized GluR2 after NMDAR activation and inhibits GluR2 recycling back to the plasma membrane.

\section{Discussion}

Previous studies have demonstrated that the regulation of the surface membrane trafficking of AMPA receptors plays an important role in synaptic plasticity (Song and Huganir, 2002; Bredt and Nicoll, 2003). In addition, recycling endosomes is suggested to supply additional

A

B

C
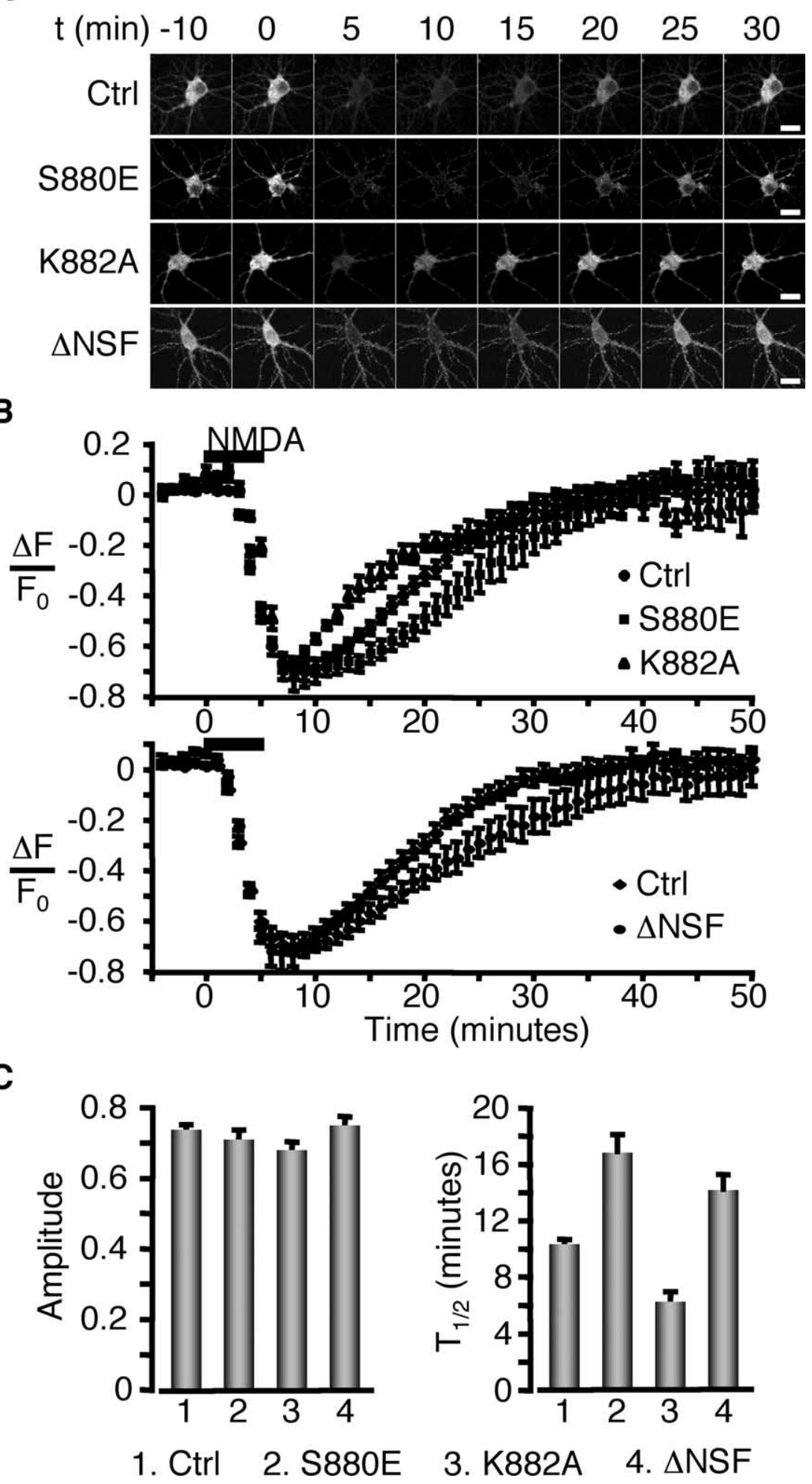

Figure 2. Modulating GluR2 5880 phosphorylation state as well as NSF binding to GluR2 alters GluR2 recycling process. $A$, Selective images of pH-GluR2 wt, S880E, K882A, and $\triangle N$ SF during NMDA perfusion/washout experiments. B, Average fluorescence time course for pH-GluR2 wt, S880E, K882A, and $\triangle$ NSF mutants during NMDA perfusion/washout experiments. C, Histograms of pHluorin fluorescence change amplitude in response to NMDA and $T_{1 / 2}$ after NMDA washout. Scale bars: $10 \mu \mathrm{m}$.

AMPA receptors during LTP induction, emphasizing the importance of the regulation of AMPAR recycling (Park et al., 2004). However, the molecular mechanisms regulating AMPAR recycling remain elusive. This is in part because of the lack of tempo- 
A

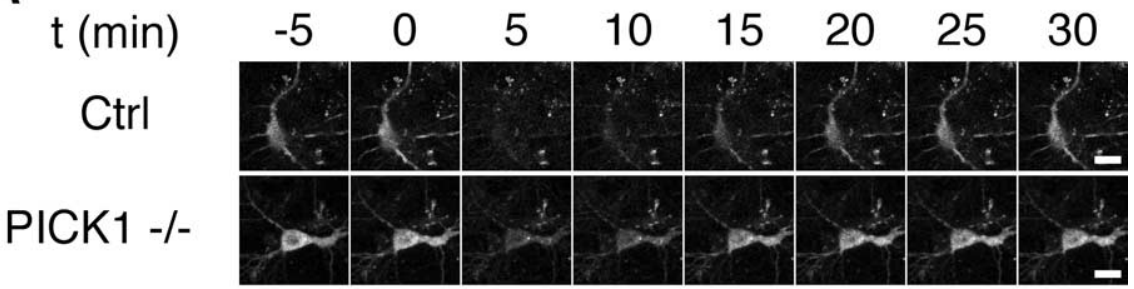

B

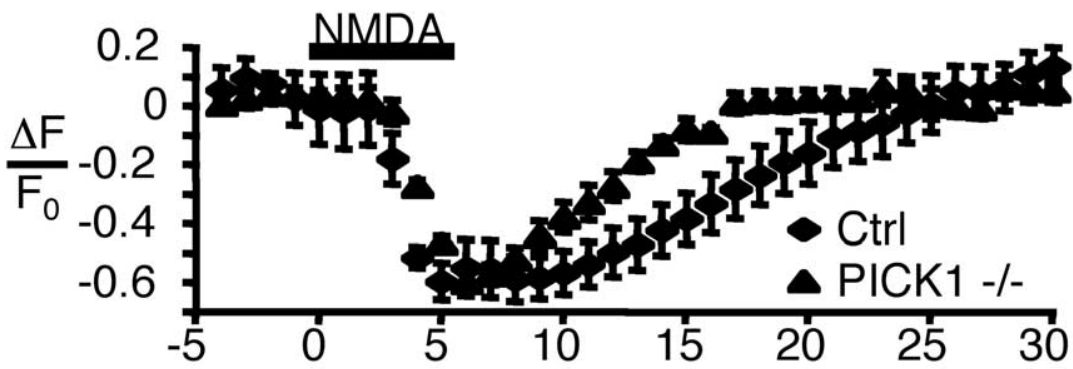

C
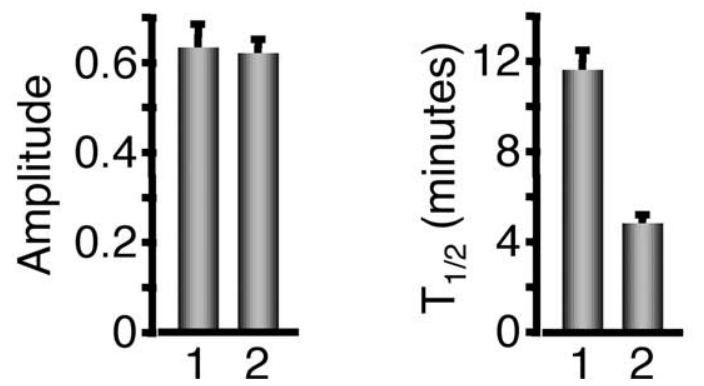

1. Ctrl

Figure 3. Neurons derived from PICK1 knock-out mice displays altered GluR2 recycling. $A$, Selective images of pH-GluR2 from wild-type and PICK1 knock-out hippocampal neurons during NMDA perfusion/washout experiments. B, Average fluorescence time course of pH-GluR2 from wild-type and PICK1 knock-out hippocampal neurons during NMDA perfusion/washout experiments. C, Histograms of pHluorin fluorescence change amplitude in response to NMDA and $T_{1 / 2}$ after NMDA washout. Scale bars: $10 \mu \mathrm{m}$.

ral resolution of existing assays for receptor recycling. Here, we used receptors tagged with ecliptic pHluorin to examine AMPAR recycling process quantitatively and with fine temporal resolution. By combining imaging pH-GluR2 and FRAP, we demonstrated that GluR2 recycles back to the surface after NMDAR activation, in agreement with early findings using cell surface biotinylation assays (Ehlers, 2000). Using this approach, we further showed that the phosphorylation state of GluR2 S880 residue as well as PICK1 binding to GluR2 regulates the rate of GluR2 recycling after NMDAR activation.

The PDZ motif at the end of GluR2 C terminus binds both PICK1 and GRIP. In addition, S880 residue within this motif is also a PKC phosphorylation site (Chung et al., 2000, 2003; Xia et al., 2000; Kim et al., 2001). In the absence of S880 phosphorylation, GluR2 preferentially binds GRIP (Chung et al., 2000; Kim et al., 2001). Phosphorylation of $\$ 880$ results in disruption of GRIP binding to GluR2 without affecting PICK1 binding (Chung et al., 2000, 2003; Kim et al., 2001). Thus, it has been suggested that the phosphorylation state of GluR2 S880 modulates GluR2 surface trafficking by regulation of GluR2 interaction with its binding partners (Song and Huganir, 2002). However, it has been difficult to examine the effect of GluR2 phosphorylation on the dynamics of receptor trafficking. In addition, it has not been clear whether PICK1 plays a direct role in GluR2 endocytosis or if it only retains
2. PICK1 -/-

internalized GluR2 intracellularly, or both. By monitoring pH-GluR2 fluorescence changes during NMDA perfusion/ washout, we found that the magnitude of internalization induced by NMDAR activation was not significantly different between pH-GluR2 wt and S880E, K882A, or $\Delta$ NSF constructs, as well as in neurons from both PICK1 knock-out mice and wild-type littermates. This suggests that neither PICK1 nor the phosphorylation of GluR2 S880 residue play a critical role in NMDAR induced GluR2 internalization process, at least in hippocampal neurons. In contrast, our data directly demonstrate that PICK1 regulates GluR2 recycling after NMDAR activation. pH-GluR2 K882A, a GluR2 mutant that cannot be phosphorylated by $\mathrm{PKC}$ and preferentially binds GRIP, displays accelerated recycling after NMDA washout. However, pH-GluR2 S880E, which mimics S880 phosphorylation and binds PICK1 but not GRIP, displays delayed recycling. In addition, $\mathrm{pH}$ GluR2 $\Delta$ NSF also demonstrates prolonged recycling. Because NSF has been shown to disassemble GluR2-PICK1 complex (Hanley et al., 2002), NSF is likely to exert its role in regulating GluR2 recycling through dissociating PICK1 from GluR2. Together, these data indicate that PICK1 favors retention of internalized GluR2 in recycling pools. Consistently, pH-GluR2 displays accelerated recycling after NMDAR activation in neurons derived from PICK1 knock-out mice. These data strongly suggest that phosphorylation of GluR2 S880 and the binding of PICK1 to GluR2 regulate the recycling of internalized GluR2. PICK1-GluR2 interaction has been shown to be important for cerebellar and hippocampal LTD, two forms of longlasting synaptic depression (Chung et al., 2000, 2003; Daw et al., 2000; Xia et al., 2000; Iwakura et al., 2001; Kim et al., 2001), as well as calcium-permeable AMPAR plasticity at cerebellar parallel fiber stellar cell synapse (Gardner et al., 2005; Liu and CullCandy, 2005). The binding of PICK1 to GluR2 is likely to regulate intracellular pools of GluR2 receptors in recycling endosomes that can be differentially regulated in different cell types in response to distinct signaling pathways.

\section{References}

Ashby MC, De La Rue SA, Ralph GS, Uney J, Collingridge GL, Henley JM (2004) Removal of AMPA receptors (AMPARs) from synapses is preceded by transient endocytosis of extrasynaptic AMPARs. J Neurosci 24:5172-5176.

Brebner K, Wong TP, Liu L, Liu Y, Campsall P, Gray S, Phelps L, Phillips AG, Wang YT (2005) Nucleus accumbens long-term depression and the expression of behavioral sensitization. Science 310:1340-1343.

Bredt DS, Nicoll RA (2003) AMPA receptor trafficking at excitatory synapses. Neuron 40:361-379.

Chung HJ, Xia J, Scannevin RH, Zhang X, Huganir RL (2000) Phosphorylation of the AMPA receptor subunit GluR2 differentially regulates its interaction with PDZ domain-containing proteins. J Neurosci 20:7258-7267.

Chung HJ, Steinberg JP, Huganir RL, Linden DJ (2003) Requirement of 
AMPA receptor GluR2 phosphorylation for cerebellar long-term depression. Science 300:1751-1755.

Daw MI, Chittajallu R, Bortolotto ZA, Dev KK, Duprat F, Henley JM, Collingridge GL, Isaac JT (2000) PDZ proteins interacting with C-terminal GluR2/3 are involved in a PKC-dependent regulation of AMPA receptors at hippocampal synapses. Neuron 28:873-886.

Dingledine R, Borges K, Bowie D, Traynelis SF (1999) The glutamate receptor ion channels. Pharmacol Rev 51:7-61.

Ehlers MD (2000) Reinsertion or degradation of AMPA receptors determined by activity-dependent endocytic sorting. Neuron 28:511-525.

Gardner SM, Takamiya K, Xia J, Suh JG, Johnson R, Yu S, Huganir RL (2005) Calcium-permeable AMPA receptor plasticity is mediated by subunitspecific interactions with PICK1 and NSF. Neuron 45:903-915.

Hanley JG, Khatri L, Hanson PI, Ziff EB (2002) NSF ATPase and alpha-/ beta-SNAPs disassemble the AMPA receptor-PICK1 complex. Neuron 34:53-67.

Hollmann M, Heinemann S (1994) Cloned glutamate receptors. Annu Rev Neurosci 17:31-108.

Huang Y, Man HY, Sekine-Aizawa Y, Han Y, Juluri K, Luo H, Cheah J, Lowenstein C, Huganir RL, Snyder SH (2005) S-nitrosylation of $\mathrm{N}$-ethylmaleimide sensitive factor mediates surface expression of AMPA receptors. Neuron 46:533-540.

Iwakura Y, Nagano T, Kawamura M, Horikawa H, Ibaraki K, Takei N, Nawa H (2001) N-methyl-D-aspartate-induced alpha-amino-3-hydroxy-5methyl-4-isoxazoleproprionic acid (AMPA) receptor downregulation involves interaction of the carboxyl terminus of GluR2/3 with Pick1. Ligand-binding studies using Sindbis vectors carrying AMPA receptor decoys. J Biol Chem 276:40025-40032.

Kim CH, Chung HJ, Lee HK, Huganir RL (2001) Interaction of the AMPA receptor subunit GluR2/3 with PDZ domains regulates hippocampal long-term depression. Proc Natl Acad Sci USA 98:11725-11730.

Kopec CD, Li B, Wei W, Boehm J, Malinow R (2006) Glutamate receptor exocytosis and spine enlargement during chemically induced long-term potentiation. J Neurosci 26:2000-2009.

Liu SJ, Cull-Candy SG (2005) Subunit interaction with PICK and GRIP controls $\mathrm{Ca}^{2+}$ permeability of AMPARs at cerebellar synapses. Nat Neurosci 8:768-775.

Miesenbock G, De Angelis DA, Rothman JE (1998) Visualizing secretion and synaptic transmission with $\mathrm{pH}$-sensitive green fluorescent proteins. Nature 394:192-195.

Nishimune A, Isaac JT, Molnar E, Noel J, Nash SR, Tagaya M, Collingridge GL, Nakanishi S, Henley JM (1998) NSF binding to GluR2 regulates synaptic transmission. Neuron 21:87-97.

Noel J, Ralph GS, Pickard L, Williams J, Molnar E, Uney JB, Collingridge GL, Henley JM (1999) Surface expression of AMPA receptors in hippocampal neurons is regulated by an NSF-dependent mechanism. Neuron 23:365-376.

Park M, Penick EC, Edwards JG, Kauer JA, Ehlers MD (2004) Recycling endosomes supply AMPA receptors for LTP. Science 305:1972-1975.

Perez JL, Khatri L, Chang C, Srivastava S, Osten P, Ziff EB (2001) PICK1 targets activated protein kinase Calpha to AMPA receptor clusters in spines of hippocampal neurons and reduces surface levels of the AMPAtype glutamate receptor subunit 2. J Neurosci 21:5417-5428.

Seidenman KJ, Steinberg JP, Huganir R, Malinow R (2003) Glutamate receptor subunit 2 Serine 880 phosphorylation modulates synaptic transmission and mediates plasticity in CA1 pyramidal cells. J Neurosci 23:9220-9228.

Song I, Huganir RL (2002) Regulation of AMPA receptors during synaptic plasticity. Trends Neurosci 25:578-588.

Song I, Kamboj S, Xia J, Dong H, Liao D, Huganir RL (1998) Interaction of the N-ethylmaleimide-sensitive factor with AMPA receptors. Neuron 21:393-400.

Steinberg JP, Huganir RL, Linden DJ (2004) N-ethylmaleimide-sensitive factor is required for the synaptic incorporation and removal of AMPA receptors during cerebellar long-term depression. Proc Natl Acad Sci USA 101:18212-18216.

Steinberg JP, Takamiya K, Shen Y, Xia J, Rubio ME, Yu S, Jin W, Thomas GM, Linden DJ, Huganir RL (2006) Targeted in vivo mutations of the AMPA receptor subunit GluR2 and its interacting protein PICK1 eliminate cerebellar long-term depression. Neuron 49:845-860.

Terashima A, Cotton L, Dev KK, Meyer G, Zaman S, Duprat F, Henley JM, Collingridge GL, Isaac JT (2004) Regulation of synaptic strength and AMPA receptor subunit composition by PICK1. J Neurosci 24:5381-5390.

Xia J, Chung HJ, Wihler C, Huganir RL, Linden DJ (2000) Cerebellar longterm depression requires PKC-regulated interactions between GluR2/3 and PDZ domain-containing proteins. Neuron 28:499-510. 